\title{
Knowledge, Awareness, and Attitudes Toward Obstructive Sleep Apnea among the Population of the Asir Region of Saudi Arabia in 2019
}

Ali M. Alshehri ${ }^{1}$, Mohammed S. Alshehri ${ }^{2}$, Omar M. Alamri ${ }^{2}$, Fayez S. Alshehri ${ }^{3}$, Mazen Alshahrani ${ }^{2}$, Mohammed A. Alflan ${ }^{2,4}$, Meshary S. Alshahrani ${ }^{2}$

1. Medicine, King Khalid University, Al-Namas, SAU 2. Medicine, King Khalid University, Khamis Mushait, SAU 3. Medicine, King Khalid University, Abha, SAU 4. Internal Medicine, Armed Forces Hospitals Southern Region, Khamis Mushait, SAU

Corresponding author: Meshary S. Alshahrani, mesharymerfaa@gmail.com

\section{Abstract \\ Background}

Obstructive sleep apnea (OSA) is a sleep disorder that involves the cessation or significant decrease in airflow along with a distinct effort to breathe. While there are several types of sleep apnea, OSA is the most common.

\section{Methodology}

This descriptive online cross-sectional study assessed knowledge, awareness, and attitudes towards OSA over 30 days in a nonrandomized sample of the general population of the Asir region of Saudi Arabia. Subjects were included if they lived in the Asir region and were aged $>18$ years.

\section{Results}

Of the respondents who participated in the study, $64 \%$ were aware of OSA, whereas $36 \%$ were not. Most respondents reported that OSA was dangerous, whereas $24 \%$ did not know whether OSA was dangerous. Moreover, $81 \%$ of respondents reported that they did not know about methods of diagnosing OSA, and 84\% did not know about the methods to treat OSA.

\section{Conclusions}

Subjects living in the Asir region of Saudi Arabia showed a low level of awareness of all aspects of OSA. Their lack of sources of knowledge indicates the need for medical staff to improve awareness and knowledge of OSA.

Received 12/20/2019

Review began 03/02/2020 Review ended 03/08/2020 Published 03/13/2020

(c) Copyright 2020 Alshehri et al. This is an open access article distributed under the terms of the Creative Commons Attribution License CC-BY 4.0 , which permits unrestricted use, distribution, and reproduction in any medium, provided the original author and source are credited.
Categories: Otolaryngology, Public Health

Keywords: knowledge, awareness, obstructive sleep apnea, asir region, saudi arabia

\section{Introduction}

Obstructive sleep apnea (OSA), the most common type of sleep apnea, is characterized by repeated episodes of complete or partial obstruction of the upper airways during sleep, despite efforts to breathe, and is associated with a reduction in blood oxygen saturation. OSA associated with excessive daytime sleepiness is commonly called OSA syndrome or OSA-hypopnea syndrome [1]. The recurrent episodes of upper airway collapse during sleep in subjects with OSA are associated with recurrent oxyhemoglobin desaturation and arousals from sleep [1].

Signs and symptoms of OSA include unexplained daytime sleepiness, restless sleep, and loud snoring (with periods of silence followed by gasps). Less common symptoms are morning headaches; insomnia; trouble concentrating; mood changes such as irritability, anxiety, and depression; forgetfulness; increased heart rate and/or blood pressure; decreased sex drive; unexplained weight gain; and increased urination and/or nocturia, and heavy night sweats. Awakenings in subjects with OSA are usually too brief for subjects to remember. Awakenings are frequently accompanied by shortness of breath, which corrects itself quickly within one or two deep breaths. Subjects may also make snorting, choking, or gasping sounds. These patterns can repeat five to more than 30 times per hour throughout the entire night [2]. These disruptions may impair subjects' abilities to reach the desired deep, restful phases of sleep, with subjects likely feeling sleepy during waking hours. Subjects with OSA may be unaware that their sleep was interrupted, with many of these subjects thinking that they slept well throughout the night [3].

Diagnosis and treatment of OSA depend on subjects' knowledge, awareness, and attitudes toward this 


\section{Cureus}

condition. The present study, therefore, evaluated the knowledge, awareness, and attitudes toward OSA in persons living in the Asir region of Saudi Arabia. This study also assessed risk factors associated with OSA in this population, including obesity, hypertension, smoking, and family history; the main sources of information from which the public obtains information about sleep and how to improve it; and attitudes toward OSA, including its complications and awareness of the seriousness of these complications.

\section{Materials And Methods}

This self-funded descriptive online cross-sectional study involved a nonrandomized sample of the general population of the Asir region of Saudi Arabia. Questionnaires were administered online over 30 days to subjects living in the Asir region who were aged $>18$ years. The validated questionnaire survey was sent to social media sites used by the Saudi population, including Twitter, Facebook, WhatsApp, Telegram, and Snapchat, and conducted online via Google survey. The questionnaire included demographic aspects such as age, gender, and level of education, as well as several health-related characteristics, such as health status, body mass index, physical activity, and smoking.

The raw data were recorded on appropriate designed excel spreadsheets and processed in accordance with the best practices for raw data management to identify any inaccuracies prior to statistical analysis. Outlying data were flagged. Categorical variables were treated similarly to identify any potential anomalies. All identified anomalies were discussed with the biostatistics team and were corrected prior to initial statistical analysis. Descriptive statistics were recorded as numbers, percentages, means, and standard deviations.

The study protocol was approved by the ethics review board of our institution, and all participants provided written informed consent. Subjects were informed of their right to withdraw from the study at any time without any obligation toward the study team. The data were collected and used for research purposes only, with only the principal investigator having access to the data. Confidentiality was maintained throughout the study by keeping the participants' identity anonymous, with the names and personal information of participants not obtained for ethical reasons.

\section{Results}

The study sample consisted of 626 participants. Of these, $37 \%$ were aged $\geqslant 45$ years, $27 \%$ were aged $36-45$ years, $20 \%$ were aged $26-35$ years, and $16 \%$ were aged $18-25$ years (Table 1 ). Slightly less than two-thirds of respondents were males (61\%), while $39 \%$ of participants were females. Analysis of respondent education level showed that most subjects were highly educated, with $64 \%$ having a bachelor`s degree, $6 \%$ having a master's degree, and 6\% having a Ph.D. In addition, $20 \%$ of participants had completed secondary school, whereas only $2 \%$ completed intermediate schools, and $1 \%$ completed primary schools (Table 2 ). Moreover, $28 \%$ of respondents worked in the healthcare field, whereas $72 \%$ did not.

\begin{tabular}{|c|c|c|}
\hline & Number & Percent \\
\hline 18-25 & 102 & 16.3 \\
\hline 26-35 & 124 & 19.8 \\
\hline $36-45$ & 171 & 27.3 \\
\hline $45+$ & 229 & 36.6 \\
\hline Total & 626 & $100.0 \%$ \\
\hline
\end{tabular}

TABLE 1: Age distribution of the participants in the study 


\section{Cureus}

\begin{tabular}{|c|c|c|}
\hline & Number & Percent \\
\hline Primary & 6 & 1.0 \\
\hline Intermediate & 15 & 2.4 \\
\hline Secondary & 124 & 19.8 \\
\hline University (Bachelor) & 401 & 64.1 \\
\hline Master & 40 & 6.4 \\
\hline PhD & 40 & 6.4 \\
\hline Total & 626 & $100.0 \%$ \\
\hline
\end{tabular}

\section{TABLE 2: Educational level of respondents}

The most frequent chronic diseases in our study respondents were diabetes mellitus (12\%), hypertension (10\%), vision problems (9\%), and arthritis (7\%), with 6\% having other chronic diseases (Table 3). Most respondents (62\%) were in good health, reporting no chronic diseases.

\begin{tabular}{|c|c|c|}
\hline & Number & Percent \\
\hline Diabetes mellitus & 73 & 11.7 \\
\hline Hypertension & 60 & 9.6 \\
\hline High cholesterol & 2 & 0.3 \\
\hline Depression & 14 & 2.2 \\
\hline Heart disease & 15 & 2.4 \\
\hline Arthritis & 45 & 7.2 \\
\hline Vision problems & 57 & 9.1 \\
\hline Respiratory diseases & 30 & 4.8 \\
\hline Cancer & 1 & 0.2 \\
\hline Others & 39 & 6.2 \\
\hline None & 389 & 62.1 \\
\hline Total & 626 & \\
\hline
\end{tabular}

TABLE 3: Percentages of participants with chronic diseases

Most subjects (54\%) reported sleeping for four to six hours per day, and $41 \%$ reported sleeping for seven to nine hours per day. Only 3\% reported sleeping for more than nine hours per day, whereas $2 \%$ reported sleeping for three or fewer hours per day. Assessments of participants' knowledge about the number of hours of sleep needed per day showed that the majority (82\%) regarded that sleep for seven to nine hours was needed, with $14 \%$ reporting that four to six hours per day were needed (Table 4 ). Only $4 \%$ reported that sleep for more than nine hours per day was needed, and only $0.2 \%$ reported that with three or fewer hours were required. About $70 \%$ of the respondents reported that the time they slept was sufficient for performing daily activities without difficulties, whereas 30\% did not. Assessment of participants' knowledge of the most important benefits of adequate sleep found that $51 \%$ regarded physical benefits, $41 \%$ enhanced thinking, $5 \%$ reported emotional benefits, and $3 \%$ regarded social benefits as most important. 


\section{Cureus}

Hours of sleep needed per day

Time (hours)

Number

Percent

$\leq 3$

1

0.2

4-6

87

7-9

514

82.1

$9+$

24

3.8

Sleep time is sufficient for performing daily activities without difficulties

Yes

Most important benefits of adequate sleep

Physical benefits

Enhanced thinking

Social benefits

Lack of sleep affects work

Yes

Drowsiness during the day

Yes

Nap during the day

Nap during the day affects sleep at night

Relationship between sleep and aging

Yes

\section{TABLE 4: Participants' knowledge of sleep and need to sleep}

Almost all respondents (98\%) believed that lack of sleep affected their work, whereas 60\% reported drowsiness during the day. Although $75 \%$ reported taking a nap during the day, $51 \%$ were aware that naps during the day affect sleep at night. In addition, $71 \%$ of respondents reposted knowledge of a relationship between sleep and aging, whereas $22 \%$ were unaware of this relationship.

Of the study participants, $64 \%$ had heard of OSA, whereas $36 \%$ had not. About $75 \%$ regarded OSA as dangerous, whereas $24 \%$ did not know whether it is dangerous or not. Moreover, $81 \%$ of respondents reported never hearing about methods of diagnosing OSA, with $84 \%$ being unaware of methods used to treat 
OSA. The majority of respondents (96\%) had never been diagnosed with OSA, with $80 \%$ regarding the diagnosis and treatment of sleep apnea as important.

\section{Discussion}

This cross-sectional study was undertaken to determine the knowledge, awareness, and attitudes toward OSA among the general population of the Asir region of Saudi Arabia. Investigations of the presence or absence of risk factors for OSA (e.g., obesity, hypertension, smoking, family history), the main sources of information to the public about sleep and how to improve it, general attitudes toward OSA and its complications, and public awareness of the seriousness of these complications may help in the diagnosis and treatment of OSA.

Many studies worldwide have assessed awareness and knowledge of OSA, with one study in China reporting that $21.5 \%$ of the respondents were aware of OSA. A total of 77 (5.9\%), 158 (12.1\%), 150 (11.5\%), and 110 (8.4\%) respondents were able to correctly list at least one risk factor, symptom, health consequence, and treatment options for OSA, respectively [4]. Among the study participants of the Asir region of Saudi Arabia, $64 \%$ had heard of OSA; $93 \%, 89 \%, 73 \%$, and $16 \%$ answers were correct regarding risk factors, symptoms, health consequence, and treatment options for OSA; the population education level of the first research showed $9.3 \%$ with no former education, $36.4 \%$ with secondary and below education, and $54.2 \%$ with postsecondary education. However, in this research, the education level showed secondary and post-secondary more than $95 \%$.

The reason for the population to seek healthcare services is their understanding and knowledge regarding the symptoms and the complications; a study involving the general population in the Lorraine region of France showed despite encouraging results regarding OSA symptoms, the general population showed limited awareness of its complications. Innovative educational campaigns must be organized to inform practitioners and the general public about the disease and raise awareness of its complications [5]. A study in the USA found that OSA is a serious illness affecting about $12 \%$ of adults, with most of these patients being undiagnosed, posing serious healthcare and economic burdens. The STOP-BANG score (Snoring, Tiredness, Observed apnea, blood Pressure, Body mass index, Age, Neck circumference, and Gender) was reported to be a reliable screening tool to identify patients with probable OSA [6]. Early diagnosis by in-lab polysomnography or home sleep apnea test should be followed by an appropriate therapeutic modality. Currently, continuous positive airway pressure therapy remains the treatment of choice for patients with moderate and severe OSA.

The importance of lifestyle modifications such as smoking cessation, weight reduction, and physical activity found to be a way of treatment. Controlled intervention trials strongly suggest that weight reduction together with a healthy diet and increased physical activity may correct or at least improve the symptoms of OSA [7].

Knowledge sources about OSA are very important. In this study, we found (26\%) of respondents seeking the doctors for information, while (23\%) they seek it from social media. Research done about the quality of YouTube as a source of information showed that YouTube is a promising source of information for OSA patients. Educational and news videos are of the highest quality. General quality measures like search position, views, and likes are not correlated with formally scored value. Sleep surgery and otolaryngologists are minimally mentioned, representing an opportunity for improvement [8].

\section{Conclusions}

The present study showed a poor level of awareness regarding all aspects of OSA among the general population of the Asir region of Saudi Arabia. Most subjects lacked sources of knowledge about this condition, indicating the need for medical personnel to improve awareness. Health education campaigns about OSA and its importance are needed, as is the training of primary healthcare physicians to raise awareness in their communities. Additional studies are also needed to assess the level of community awareness about OSA and its complications.

\section{Additional Information \\ Disclosures}

Human subjects: Consent was obtained by all participants in this study. Animal subjects: All authors have confirmed that this study did not involve animal subjects or tissue. Conflicts of interest: In compliance with the ICMJE uniform disclosure form, all authors declare the following: Payment/services info: All authors have declared that no financial support was received from any organization for the submitted work. Financial relationships: All authors have declared that they have no financial relationships at present or within the previous three years with any organizations that might have an interest in the submitted work. Other relationships: All authors have declared that there are no other relationships or activities that could appear to have influenced the submitted work. 


\section{Cureus}

\section{References}

1. Guilleminault C, Tilkian A, Dement WC: The sleep apnea syndromes. Annu Rev Med. 1976, 27:465-484. 10.1146/annurev.me.27.020176.002341

2. Sleep apnea. National Heart, Lung, and Blood Institute. (2019). Accessed: April 2, 2019: http://www.nhlbi.nih.gov/health-topics/sleep-apnea.

3. Bonsignore MR, Baiamonte P, Mazzuca E, Castrogiovanni A, Marrone O: Obstructive sleep apnea and comorbidities: a dangerous liaison. Multidiscip Respir Med. 2019, 14:8. 10.1186/s40248-019-0172-9

4. Sia CH, Hong Y, Tan LWL, van Dam RM, Lee CH, Tan A: Awareness and knowledge of obstructive sleep apnea among the general population. Sleep Med. 2017, 36:10-17. 10.1016/j.sleep.2017.03.030

5. Arous F, Boivin JM, Chaouat A, Rumeau C, Jankowski R, Nguyen DT: Awareness of obstructive sleep apneahypopnea syndrome among the general population of the Lorraine Region of France. Eur Ann Otorhinolaryngol Head Neck Dis. 2017, 134:303-308. 10.1016/j.anorl.2017.02.010

6. Munish Goyal, Jeremy Johnson: Obstructive sleep apnea diagnosis and management. Mo Med. 2017, 114:120-124.

7. Tuomilehto H1, Seppä J, Uusitupa M: Obesity and obstructive sleep apnea--clinical significance of weight loss. Sleep Med Rev. 2013, 17:321-9. 10.1016/j.smrv.2012.08.002

8. Singh SK, Liu S, Capasso R, Kern RC, Gouveia CJ: YouTube as a source of information for obstructive sleep apnea. Am J Otolaryngol. 2018, 39:378-382. 